\title{
ANALYSIS OF INTERFERENCE AND CHANEL CAPACITY IN A CDMA WIRELESS NETWORK Using Dynamic Channel Assignment (DCA) STRATEGY
}

\author{
${ }^{1}$ Ohaneme C.O., ${ }^{2}$ Idigo V.E., ${ }^{3}$ Nnebe S.U. and ${ }^{4}$ Ifeagwu E.N. \\ 1, 2, 3,4 Department of Electronic and Computer Engineering, Nnamdi Azikiwe \\ University, Awka, Nigeria. \\ ${ }^{1}$ engrohaneme@yahoo.com, ${ }^{2}$ viceze2006@yahoo.com, \\ ${ }^{3}$ scholar.nnebe@gmail.com, ${ }^{4}$ skotolysis@yahoo.com
}

\begin{abstract}
There had been astronomical increase in the number of mobile users in recent times. Investigation had shown that these increased numbers of mobile users contribute high percentage of interferences that degrades the Quality of Services (QoS) in wireless network. Hence, interference is the most limiting factor of improved capacity in CDMA cellular network and has been one of the problems militating against the high efficiency of any mobile network. In effect, the scarcity of radio frequency spectrum and the ever increasing network users has necessitated the need to adopt some channel assignment algorithm to achieve acceptable spectrum efficiency. This paper therefore analyses interferences in a CDMA-based DCA algorithm with special emphasis placed on Adjacent Channel Interference (ACI) and Co-Channel Interference (CCI). The CDMA system used in this study is Zoom, one of the leading mobile wireless networks in South-East Nigeria. The developed models are simulated using Matlab. It was therefore discovered that interferences have prominent roles to play in reliable channel assignment strategy and network capacity in wireless system. Hence, in this paper, any reduction in interferences in cellular systems translates into improving channel capacity of a wireless network.
\end{abstract}

Keywords: Interference, DCA, cellular network, co-channel interference, adjacent channel interference, downlink, uplink.

\section{Introduction}

The scarcity of radio spectrum in wireless network and the ever increase in the network users leaves the communication channels so crowded. This introduces interference in the entire communication system thereby degrading the Quality of Service (QoS) and system capacity. Therefore there is the need to examine the possible options for increasing the capacity of the available radio frequency by reducing interferences that exist in the system. This led to the advent of the multi-user access and channel assignment technologies. Both techniques are aimed at maximizing the available radio frequency spectrum. CDMA came to the lime light as a result of the need to accommodate the requirements of the third generation (3G) wireless system [1] in which high quality data, throughput, multimedia, streaming audio, streaming video and broadcast-type services to users are supported. CDMA, being an interference limited mode of access technology system utilizes its ability to provide the entire available spectrum for communication to its teaming users as an improvement to the existing TDMA system. The interference limited nature of CDMA scheme in the entire cellular system as a result of large 
International Journal of Computer Networks \& Communications (IJCNC) Vol.4, No.5, September 2012 number of users accessing the network simultaneously need much to be desired in terms of network capacity improvement.

In this work, special emphasis is placed on the analysis of adjacent Channel Interference (ACI) for different cell layouts. It is aimed at demonstrating the effect of ACI on CDMA based DCA systems. This work also investigates the influence of Adjacent Channel Interference (ACI) and co-channel Interference (CCI) on CDMA based wireless systems. This explains in details the benefits of DCA over other assignment techniques and why CDMA is being interwoven with DCA technique. The existing cellular system deploys FCA amongst other channel assignment schemes. Channel Assignment deals with the allocation of channels to cells in a cellular network [2]. Once the channels are allocated, cells may then allow users within the cell to communicate via the available channels. Channels in a wireless communication system typically consist of time slots, frequency bands and/or CDMA pseudo noise sequences, but in an abstract sense, they can represent any generic transmission resource. There are three major categories of assigning these channels to cells (or base-stations). They include Fixed Channel Assignment (FCA), Dynamic channel Assignment (DCA), and Hybrid Channel Assignment (HCA) which is a combination of the first two methods.

In FCA, each channel is assigned to any intending user in a semi-permanent basis. This means that the user is dedicated to the channel as long as the user exists in the network. Fixed channel assignment systems allocate specific channels to specific cells. The problem with FCA system is its inability to adapt to traffic changes as most often wireless networks do not have uniform traffic. Consider a case in which two adjacent cells are allocated $\mathrm{N}$ channels each. There clearly can be situations in which one cell has a need for $\mathrm{N}+\mathrm{K}$ channels while the adjacent cell only require N-M channels (for positive integers of $\mathrm{K}$ and $\mathrm{M}$ ). In such a case, $\mathrm{K}$ users in the first cell would be blocked from making calls while $M$ channels in the second cell would go unused. Clearly in this situation of non-uniform spatial offered traffic, the available channels are being used inefficiently. FCA has been implemented on a widespread level to date.

Dynamic Channel Assignment (DCA) attempts to alleviate the problem mentioned for FCA systems when offered traffic is non-uniform. In DCA systems, channels for the entire network are kept in a pool of resources so that whenever a channel is needed by a cell, the channel is allocated under the constraint that frequency reuse requirements cannot be violated [3]. One of the problems associated with DCA is that DCA methods often involve complex algorithm for deciding which available channel is most efficient. These algorithms can be very computationally intensive and may require large computing resources in order to be real-time.

The third category of channel assignment techniques is the hybrid of fixed and dynamic channel allocation systems. Several methods have been presented that fall within this category and in addition, a great deal of comparison has been made with corresponding simulations and analysis [4].

However, in this work, the mobile telephone switching office (MTSO) also known as Mobile Switching Center (MSC) of Zoom mobile Nigeria located at PortHarcourt, Nigeria, is used as the test- bed from where the measured data deployed in the analysis were obtained. The data obtained are shown in Table 1.

This paper is structured in the following ways: Section 2 summarizes some of the related works to the subject matter. Section 3 gives brief explanation of wireless mobile communication system under study. Section 4 specifies the interference and capacity of the wireless system, where the effect of interferences on both the uplink and downlink is analyzed. Section 5 discusses the results obtained after the simulation while section 6 draws the conclusion of the work. 


\section{Related Works}

There had been several works on the improvement of wireless network capacity through various techniques. The related literatures presented in this work represent the various techniques that have been deployed to enhance mobile system capacity at various locations. [5], in their works, proposed the use of concentric circle geometry in the estimation of the capacity of multi-cellular CDMA system. They analyzed the system by asserting that once the interference effects of a single cell by the number of cells within the surrounding layer is specified, one can simply multiply the effects of a single cell by the number of cells within the geographical area. However, this technique tends to assume that all the cells that cover the geographical area are homogenous and of similar characteristics, which is not always the case. Hence, though this technique provided a good analysis of the improved system, it cannot be applied in heterogeneous areas, where cells do not have similar characteristics and properties. In the investigation of interference on CDMA network, [6] analyzed the effect of adjacent channel interference (ACI) on capacity in a hybrid TDMA/CDMA system using Time Division Duplex (TDD). Statistical approach on user distribution was used in the investigation. The results obtained show that the level of improvement in the system capacity is dependent on the number of cells in the cell clusters within the area as it describes the number of ACI, which degrades the service quality. Besides, [7] summarized the effect of interference on cellular system by measuring the Carrier-to-Interference (C/I) levels of the system independently. This helped to specify the level of interference and its effect on signal quality of wireless network. [8] analyzed the effect of other cell (inter-cell) interference on the capacity of wireless network with special emphasis on the adjacent channel interference (ACI). The authors deployed power control technique in the reverse channel to reduce the interference in the system thereby increasing the channel capacity. A strategy to remove the interference while allowing different users to transmit and receive simultaneously was proposed by [9]. The utilized MIMO wireless system with $\mathrm{Z}$ interference channel in their work provided the necessary platform to showcase how interferences can be removed to increase the number of users by improving the performance of the system.

\section{Wireless Mobile Communication System}

For certain types of devices, the aim is to achieve full spatial coverage. Generally, in conventional wireless systems, a mobile entity is linked to the base station (BS). BSs are connected to a radio network controller which uses additional interfaces that cater for the access to the public switched telephone network (PSTN). The principle structure of a cellular wireless system is shown in figure 1 .

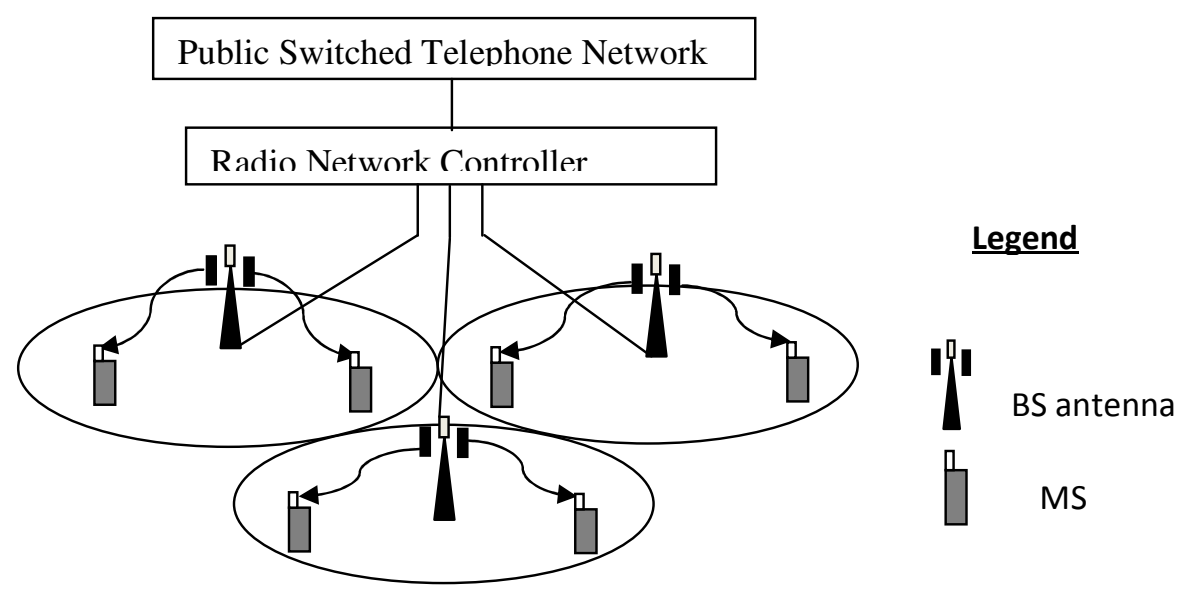

Figure 1: A cellular Wireless Network 


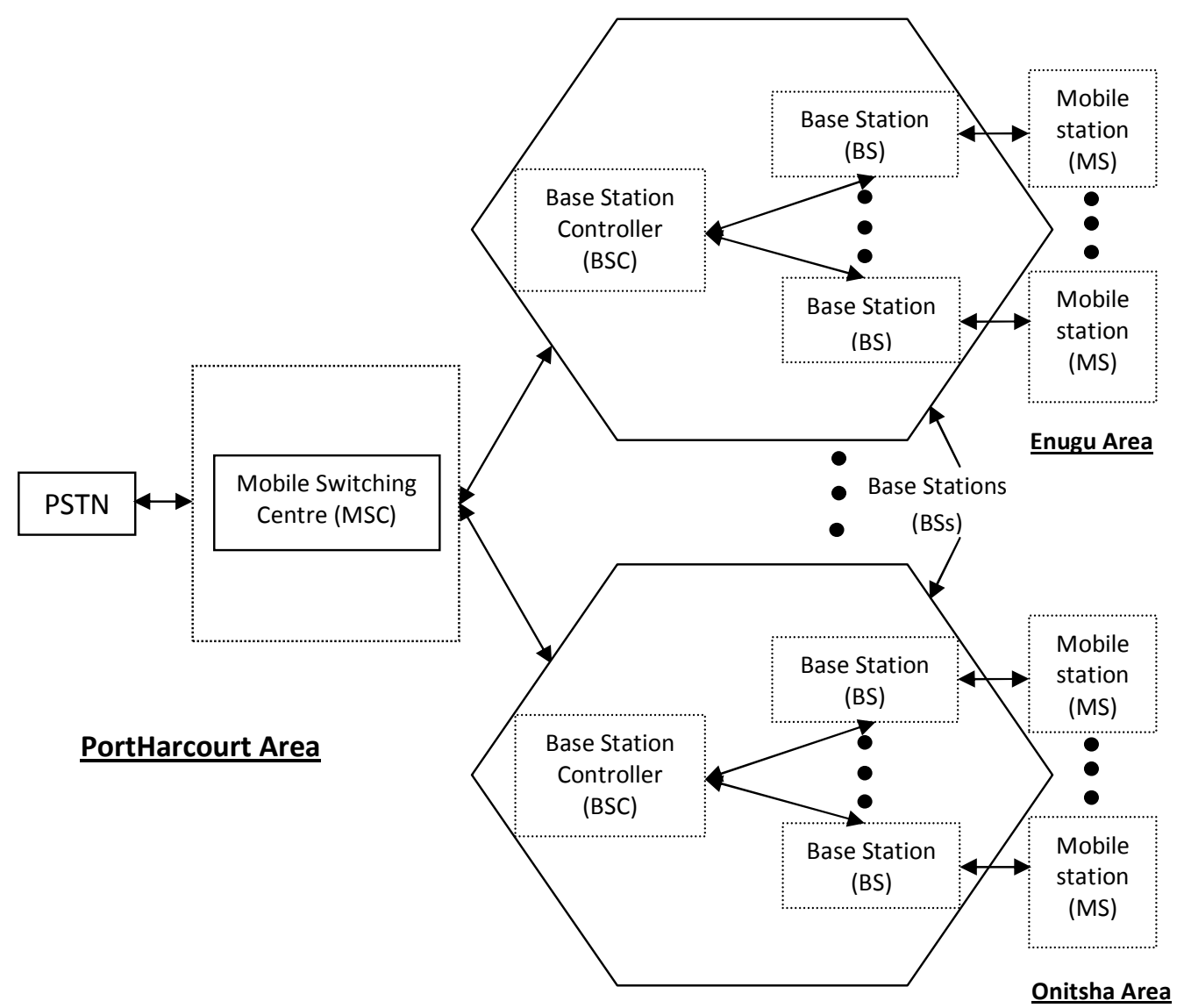

Figure 2: The Wireless Network coverage of Zoom, South-East Nigeria

Since the total available radio resource is limited, the spatial dimension is used to allow wide area coverage. This is achieved by splitting the radio resource into channels (groups of frequencies). These groups are then assigned to different contiguous cells. This pattern is repeated as often as necessary until the entire area is covered. A single pattern is equivalent to a cluster. Therefore, a radio resource which is split into $\boldsymbol{i}$ groups directly corresponds to a cell cluster of size $\boldsymbol{i}$. In this way, it is ensured that the same radio resource is only used in cells that are separated by a defined minimum distance called reuse distance. As a consequence, the separation distance grows if the cluster size increases. The frequency (co-channel) reuse ratio $q$ from geometry of hexagons [10] gives

$$
q=\frac{D}{R}=
$$

and

$$
k=i^{2}+i j+j^{2}
$$

where $\mathrm{D}$ is the separation distance (re-use distance) and $R$ is the radius of the cell, $k$ is the cluster size while the values of $i$ and $j$ (integers) are the normalised distance between centres of adjacent and co-channel cells. Then the density $(\boldsymbol{\rho})$ of the channels can be represented as

$$
\rho=\frac{B_{w}}{A_{c} B}
$$


International Journal of Computer Networks \& Communications (IJCNC) Vol.4, No.5, September 2012

where $A_{c}$ is the area of the cell and $\frac{B_{w}}{B}$ is the number of channels and $B_{w}$ is the total band width of the cell and $B$ is the bandwidth of each user. Hence, increasing the cluster size acts in favour of low interference. However, an increased cluster size means that the same radio resource is used less often within a given area. As a result, fewer users per unit area can be served. Therefore, there is a trade-off between cluster size and capacity. In an ideal scenario, the total available radio resource would be used in every cell while the interference is being kept at a tolerable level.

\section{Interference and Channel Capacity of a CDMA System}

Channel capacity for a radio system can be defined as the maximum number of channels or users that can be provided in a fixed frequency band [5]. Radio capacity is a parameter which measures spectrum efficiency of a wireless system. This parameter is determined by the required Signal-to-interference ratio $\left(\frac{S}{I}\right)$ and the channel bandwidth $\left(B_{C}\right)$. In a cellular system, the interference at a base station receiver may be coming from the mobile users in the surrounding cells, (otherwise called inter-cell interference) or from mobile users within the same cell (also known as intra-cell interference). This is called Reverse Channel Interference (RCI). For a particular mobile user, the desired base station will provide the Forward Channel Interference (FCI). The capacity of CDMA system is interference limited, therefore, any reduction in the interference will cause a linear increase in the capacity of CDMA. In other words, in a CDMA system, the link performance for each user increases as the number of users decreases [7].

In actual CDMA cellular systems that employ separate forward and reverse links, neighbouring cells share some frequencies, and each base station controls the transmit power of each of its own intra-cell users. However, a particular base station is unable to control the power of users in neighbouring cells, and these users add to the noise floor and decrease capacity on the reverse link of the particular cell of interest.

The transmit powers of each inter-cell user will add to the intra-cell interference (where users are under power control) at the base station receiver. The amount of inter-cell interference determines the frequency reuse factor $f$, of a CDMA cellular system [5]. Ideally, each cell shares the same frequency and the maximum possible value of $f(f=1)$ is achieved. In practice, however, the inter-cell interference reduces f significantly.

The frequency reuse factor for a CDMA system on the reverse link is defined by [7] as

$$
f=\frac{N_{I}}{N_{I}+\sum_{i} U_{i} N_{a i}}
$$

And the frequency reuse efficiency, $F$ is defined as

$$
F=f \times 100 \%
$$

where $N_{I}$ is the total interference power received from the N-I in cell users, $U_{i}$ is the number of users in the $i$ th adjacent cell and $N_{a i}$ is the average interference power for a user located in the $i t h$ adjacent cell. 
International Journal of Computer Networks \& Communications (IJCNC) Vol.4, No.5, September 2012 Within the cell of interest, the desired user will have the same received power as the N-1 undesired in-cell users when power control is employed and the average received power from the users in an adjacent cell can be found by

$$
N_{a i}=\sum_{j} \frac{N_{i j}}{U_{i}}
$$

where $N_{i j}$ is the power received at the base station of interest from the $j t h$ user in the $i t h$ cell. Each adjacent cell have a different number of users and each inter-cell user will offer different level of interference depending on its exact transmitted power and location relative to the base station of interest. The variance of $N_{a i}$ can be computed using standard statistical techniques for a particular cell.

\subsection{The Reverse (Up) link in a CDMA System}

Ideally, in a CDMA system, all co-existing users appear as Gaussian noise. Therefore, the required signal-to-interference ratio (SIR), when assuming a multiple cell environment can be computed as follows.

$$
S I R=\frac{P_{u}}{\sum_{i \neq j}^{M} \beta_{j} P_{u i}+I_{o i}+N}
$$

Here, $P_{u}$ is the signal power of the desired user in the uplink, $P_{u i}$ is the interference power received from a MS using the same channel in the same cell. $\beta_{j}$ is the voice/data activity factor of the $j t h$ user. $I_{o i}$ is the interference power from other cells; $N$ is the thermal noise power and $M$ is the number of MSs per cell. It can be seen that the interference is composed of three parts.

Intra-cell interference: This is equivalent to multiple access interference (MAI) due to the cross correlation of the spread spectrum signals in a CDMA system. The problem of this receiver is that the complexity increases with the length of the spreading codes.

Inter-cell interference: Inter-cell interference can be divided into Co-channel interference (CCI) and Adjacent Channel Interference (ACI) conveyed by neighbouring or co-existing Cells in a cellular environment. CCI is the interference due to co-channel mobile users in the same coverage area. To reduce CCI, co-channel cells must be physically separated by a minimum distance to provide sufficient isolation due to propagation. Also interference resulting from signals which are adjacent in frequency to the desired signal is called ACI. ACI results from imperfect receiver filters which allow nearby frequencies to leak into the passband. It can be minimized through careful filtering and channel assignment.

For comparison the interference cancellation in the case of assigning the MS to the best BS yields better result in terms of improved quality of service. Given that the frequency reuse of a cellular CDMA system is generally considered to be unity, it is obvious that inter-cell interference can significantly reduce the advantages obtained by multi-user detectors [11]. This mechanism was put into a more general context using the Shannon capacity equation $[2,12]$ of a multi cell system without interference cancellation with the capacity of a multi cell system assuming perfect intra-cell interference cancellation.

The Shannon's Channel Capacity for the AWGN (Addictive White Gaussian Noise) channel is

$$
N_{c}<B \log _{2}\left(1+\frac{P}{N_{o} B}\right)
$$


International Journal of Computer Networks \& Communications (IJCNC) Vol.4, No.5, September 2012 where $N_{C}$ is the channel capacity (bits per second), $B$ is the transmission bandwidth (Hz), $\mathrm{P}$ is the received signal power $(\mathrm{W})$, and $N_{0}$ is the single-sided noise power density (W/Hz). The received power at a receiver is given as

$$
P=E_{b} R_{b}
$$

where $E_{b}$ is the average bit energy, and $R_{b}$ is the bit rate. The channel capacity can be normalized by the transmission bandwidth and is given by

$$
\frac{N_{C}}{B}=\log _{2}\left(1+\frac{P}{N_{0} B}\right)=\log _{2}\left(1+\frac{E_{b} R_{b}}{N_{0} B}\right)
$$

However, if $W$ is the total bit-rate and can be calculated as

$$
W=\sum_{i=1}^{M} R_{b}
$$

with $R_{b}$ being the bit-rate of a single user. If the bit rate of each user is the same, it holds that

$$
W=M_{a} R_{b}
$$

where $M_{a}$ is the number of simultaneously active users when assuming ideal interference cancellation. Substituting equation 11 into equation 9 and rearranging yields.

$$
\frac{M_{a}}{G_{p}}<\log _{2}\left(1+\frac{E_{b} R_{b}}{N_{o} B}\right)
$$

where $G_{p}$ is the processing gain defined as $G_{p}=B / R_{b}$. It can be stated from [8] that in the case when all cells are equally loaded, and all BSs deploy power control on their population of MSs, the inter-cell interference is proportional to $N_{C}$. Thus, the interference power from the neighbouring cells can be written as follows.

$$
I_{o i}=N_{o} B=\alpha N_{C}
$$

where $\boldsymbol{\alpha}$ is the proportionality factor described above. Equation 13 is substituted in equation 12 and the result is

$$
\frac{M_{a}}{G_{p}}<\log _{2}\left(1+\frac{1}{\alpha}\right)
$$

In order to access the impact of interference cancellation for a cellular CDMA system, the performance of ideally coded users with power control is assumed, i.e, the power received from each MS within a cell is the same (i.e., $P_{u}=P_{u 1}=P_{u 2=, \ldots \ldots . .} P_{u m}$ ). Hence, the intra-cell interference can be expresses as follows.

$$
I_{o}=\left(M_{a}-1\right) P_{u} \approx M_{a} P_{u}=P
$$

where $M_{a}$ is the number of simultaneously active MSs in the multi cell environment without interference cancellation. Using equations 13 and 15, and assuming $N$ to be negligible, equation 7 becomes.

$$
S_{I} R_{u}=\frac{P_{u}}{M_{a}\left(I_{o}+I_{o i}\right)}-\frac{1}{M_{a}(1+\alpha)}-\frac{E_{b} / N_{o}}{G_{p}}
$$


International Journal of Computer Networks \& Communications (IJCNC) Vol.4, No.5, September 2012 The bit energy-to-interference ratio is expressed by $E_{b} / N_{o}$. From equation 16 , the number of users in the multi cell environment can be found to be

$$
M_{a}=\frac{G_{p}}{\left(E_{b} / N_{o}\right)(1+\alpha)}
$$

In a AWGN channel $E_{b} / N_{o}$ has a lower bound and it is found to be

$$
\frac{E_{b}}{N_{o}}>\ln 2
$$

This bound equals Shannon capacity for a channel with infinitely wide bandwidth. Applying equation 18 into equation 17, the number of users in a multi cell environment divided by the processing gain in upper bound is found by

$$
\frac{M_{a}}{G_{p}}<\frac{1}{(1+\alpha) \ln 2}
$$

Let $A$ be the ratio of the capacity in the case of perfect interference cancellation, equation 14 and without interference cancellation, equation 19 becomes

$$
A=\frac{M}{M_{a}}=(1+\alpha) \ln \left(1+\frac{1}{\alpha}\right)
$$

\subsection{The Forward (Down) link in a CDMA Wireless System}

The main differences between the uplink and downlink are that synchronous transmission can be applied in a downlink, where as in the uplink, asynchronous transmission must be assumed [6], and each BS may transmit user specific signals and a common pilot signal for coherent demodulation. A consequence is that orthogonal codes are used (for example Walsh codes) to distinguish individual users. The orthogonality in the downlink can be maintained (no intra-cell interference) assuming that multipath propagation does not violate the orthogonality at the mobile receiver [13]. Therefore an orthogonality factor $\boldsymbol{\tau}$ is defined as

$$
\tau=\frac{E_{b}}{I_{0}}\left(\frac{E_{b}}{N_{0}}\right)^{-1}
$$

where $\frac{E_{b}}{I_{o}}$ is the bit-energy-to-interference ratio when the orthogonality is not maintained and thus, the signal is corrupted by intra-cell interference. The ratio $\frac{E_{b}}{N_{o}}$ is the bit-energy to interference ratio for the case that orthogonality is entirely maintained. From the definition of $\boldsymbol{\tau}$, it can be seen that the higher its value, the more the signals are corrupted by multipath propagation [5]. As reported that $\boldsymbol{\tau}$ may vary between 0.3 and 0.8 [13] with the greater value obtained in environments which are subject to severe multipath propagation. When an additional pilot signal is used, the total carrier power $P_{d}$ for the downlink yields

$$
P_{d}=P_{\text {pilot }}+\sum_{i=1}^{M} P_{d i}
$$

where $P_{\text {pilot }}$ is the pilot signal power and $P_{d i}$ is the carrier power for the $i$ th user. A factor $\varphi$ is used to model the user specification of the total carrier power [14].

$$
\varphi=\frac{P_{T}}{P_{d}}
$$


International Journal of Computer Networks \& Communications (IJCNC) Vol.4, No.5, September 2012 $\varphi=0.8$ is used with the approximation of

$$
\sum_{i=1}^{M-1} P_{d i}=\sum_{i=1}^{M} P_{d i}
$$

The Signal-to- interference ratio at the MS, $S I R_{d}$ (in the downlink) can be modelled as follows

$$
S I R_{d}=\frac{\frac{P_{d i}}{a_{i}}}{\frac{\tau}{\varphi a_{i}}\left(\sum_{i \neq j}^{M} P_{d j}\right)+I_{o d}+N_{o}}
$$

where $a_{i}$ is the path loss between the desired MS in $i$ cell and the respective BS. In a Severe multipath environment $(\tau=0.8)$, the advantages due to synchronous transmission may be cancelled by a greater transmitted carrier power as a consequence of the pilot signal $(\varphi=0.8)$. For this scenario $(\tau=\varphi)$, equation 25 yields

$$
S I R_{d}=\frac{\frac{P_{d i}}{a_{i}}}{\frac{1}{a_{i}}\left(\sum_{i \neq j}^{M} P_{d j}\right)+I_{o d}+N}
$$

In general,

$S I R=\frac{S}{(N-1) S+n}$

$S=$ Power of the received signal from each user, $N=$ Number of users in the cell, and $n=$ background noise. Then energy per bit-to-noise density ratio is given as

$$
\frac{E_{b}}{N_{o}}=\frac{S / R}{(N-1)^{S / w^{+n} / w}}
$$

$E_{b}$ is energy per bit seen at the receiver, $w$ is the spreading bandwidth, $R$ is information bit rate, $N$ is the noise power spectral density while $W / R$ is the processing gain $\left(P_{g}\right)$.

$$
\partial=\frac{t_{o f f}}{t_{s l o t}}
$$

where $\partial$ is frame synchronization, $t_{o f f}$ is time offset and $t_{\text {slot }}$ is time slot (TS) duration.

Using the synchronization mechanism, ACI in TDD system at an arbitrary location specified by its $\boldsymbol{x}$ and $\boldsymbol{y}$ coordinates, can be expressed as follows;

$$
I_{\partial d}(x, y)=\frac{1}{k_{1}} \sum_{j=1}^{L} \sum_{i=1}^{M_{j}} \partial_{j} \frac{P_{u i j}}{a_{i}(x, y)}+\left(1+\partial_{j}\right) \frac{P_{d j}}{a_{j}(x, y)}
$$

where $L$ is the number of neighbouring cells taken into consideration, $M_{j}$ is the total number of active users in the neighbouring cell $j, P_{u i j}$ is the transmitted carrier power of user $i$ in cell $j$, $P_{d j}$ describes the total carrier power transmitted by BS $j$ and $a_{i}(x, y)$ represents the path loss between the interfering user $I$ and the location of interest $(x, y)$. Similarly, $a_{j}(x, y)$ is the path loss between the location of interest and the BS of cell $j$. The synchronization between cell $j$ and the point of interest $(x, y)$ is expressed by $\partial_{j}$. The adjacent channel interference ratio (ACIR) is determined by two factors: (a) one related to the transmitter filter and referred to as: Adjacent Channel Leakage Ratio (ACLR), and (b) one related to the receiver filter and described as Adjacent Channel selectivity (ACS). The relationship between ACIR, ACLR and ACS was investigated by [15] and found to be: 
International Journal of Computer Networks \& Communications (IJCNC) Vol.4, No.5, September 2012

$$
K_{I}=\frac{1}{\frac{1}{K_{L}}+\frac{1}{K_{S}}}
$$

where $K_{L}$ is the ACLR and $K_{S}$ is the ACS.

The path loss model with no wall or floor losses, as can be found in [15] is:

$$
a_{i}=37+\varphi 10 \log (d)+\varepsilon(d B)
$$

where $\varphi$ is the path loss exponent and $d$ is the distance between transmitter and receiver. Lognormal shadowing is modelled by $\varepsilon$ with the standard deviation $\sigma$ and zero mean

\section{Simulation Results and Analysis}

The system simulation and analysis is performed using the mathematical relations derived in session 2. The results of the simulation are shown graphically using Matlab. The table 1 shows the simulation parameters used in analyzing the CDMA-based dynamic channel assignment system.

\begin{tabular}{|l|l|}
\hline \multicolumn{2}{|c|}{ Table 1: Simulation parameters } \\
\hline \multicolumn{1}{|c|}{ Parameters } & \multicolumn{1}{c|}{ Values } \\
\hline Tx power from the MS & $15 \mathrm{~dB}$ \\
\hline Tx power from BS & $24 \mathrm{~dB}$ \\
\hline Cell radius $(R)$ & $1.25 \mathrm{~km}$ \\
\hline BS separation distance & $2.5 \mathrm{~km}$ \\
\hline Path loss exponent $(\varphi)$ & 3.0 \\
\hline Chip rate & $3.84 \mathrm{Mcps}$ \\
\hline Bit-energy-to-interference ratio $\left(\frac{E_{b}}{I_{o}}\right)$ & \\
\hline
\end{tabular}

Source: MTSO, Zoom Mobile Portharcourt, Nigeria

\subsection{Analysis}

From equation 30, 31 and 32 a relationship can be derived between interference power from MS (mobile station), interference from BS (Base station), and total accumulated interference with the base separation distance $d_{o}$. Figure 3 shows the plot of the relationship of the interferences between the BSs with respect to separation distance between them. Also figure 4 explains the variation of ACI with distance over different scenarios of the communication environment. At these different scenarios, the frame synchronisation of between 0.01 and 0.99 (i.e., $1 \%$ and $99 \%$ ) is taken into consideration. 
International Journal of Computer Networks \& Communications (IJCNC) Vol.4, No.5, September 2012 The interference results for a user population of four MSs are shown in figures 3 and 4 . The interference caused by the BSs is shown in figure 3, where as the interference resulting from the MSs are shown in figure 4. Note that when the interfering cells completely overlap, the MS-BS interference is lowest (due to power control and high cross- correlation of lognormal shadowing between the interference and the desired path), but the BS-BS interference is highest. As the BSs are separated, the BS-BS interference is decreasing monotonically and at the same time the MS-BS interference is growing until the BS separation is about the cell radius. The reason for the peak of MS-BS interference is that the interference from MSs increases when the target BS is moved towards the cell boundary due to the high transmission powers of the MS's at outer regions of the cell. The MS-BS interference however diminishes in a single cell scenario as the cells move further apart.

When the density power and interference at BSs is considered as shown in figure 5, there is linear relationship between the thermal noise and inter-cell interferences. Thus reducing the thermal noise improves the capacity of the network. The intra-cell interference is plotted to show how it varies in relation to the relative capacity of the system (see figure 6). The capacity of a cellular CDMA system can be greater than the processing gain, in contrast to that of an FDMA or TDMA system, which is always less than or equal to the processing gain. Also as the inter-cell interference increases, the total capacity diminishes and the gain due to multiuser detection decreases significantly. Therefore in order to achieve a high cellular capacity, there is the need to minimize inter-cell interference as only this will enable the efficient use of technique such as interference cancellation. This can be achieved by maintaining the minimum reuse distance and also through power control.

Figure 7 shows how SIR varies with the number of users in the network. It shows that as the number of users increase, the SIR equally decreases lognormally.

However, a graph is plotted as shown in figure 9 showing the relationship between $E_{b} / N_{o}$ with the number of users. From the falling slope graph, it is clearly seen that $E_{b} / N_{o}$ decreases as the number of users in the system increases (i.e., between 0 and 240 users). It is seen that it has maximum value at 0 numbers of users and its minimum value at 240 users. Thus the energy that is being transmitted per bit is highly attenuated in a case where there are many users than the system can accommodate.

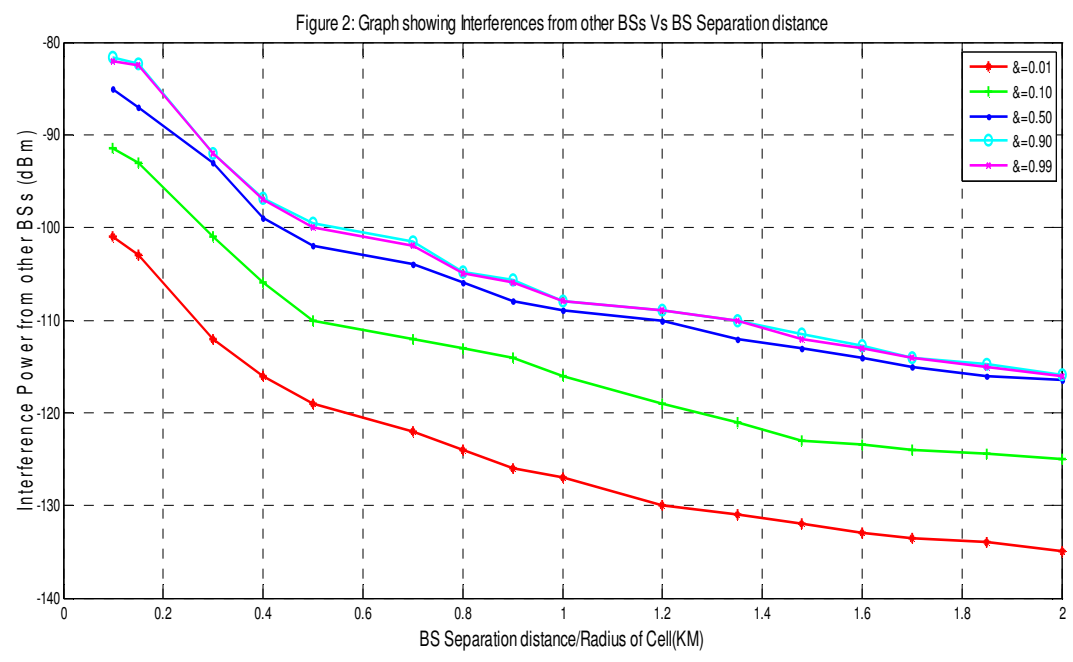

Figure 3: The relationship between interference from BS and BS separation distance 
International Journal of Computer Networks \& Communications (IJCNC) Vol.4, No.5, September 2012

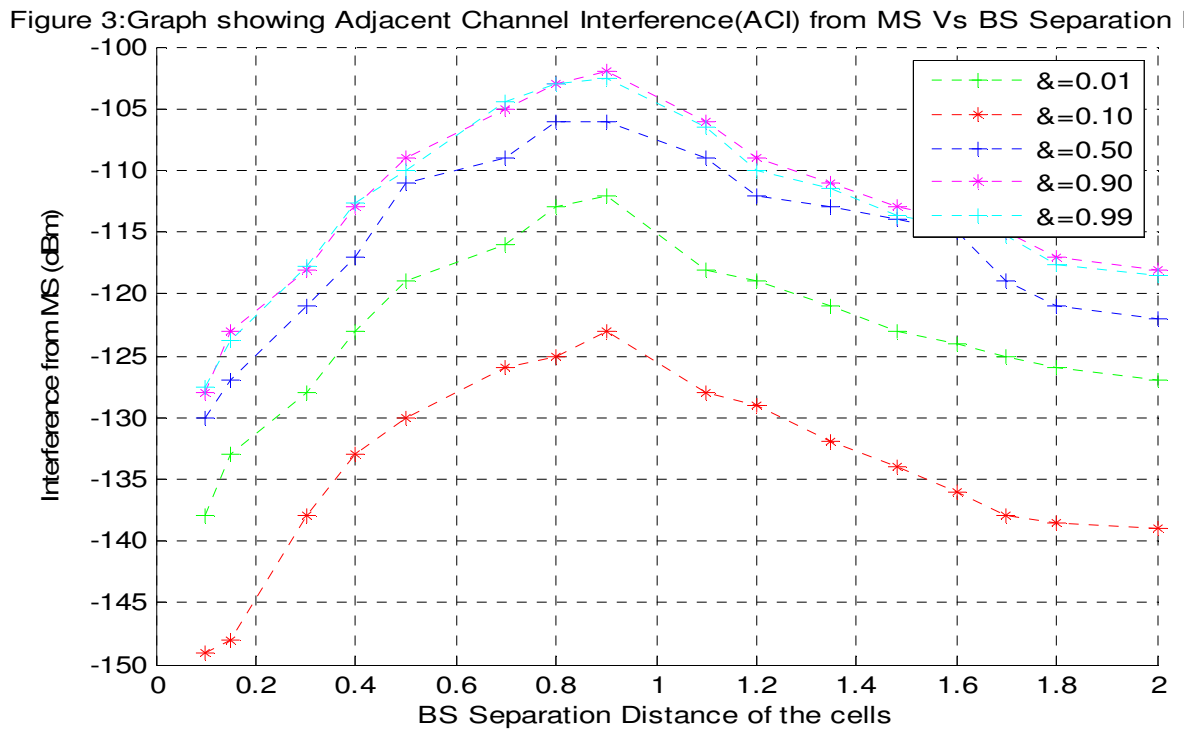

Figure 4 Adjacent Channel Interference (ACI) from MS vs BS separation distance

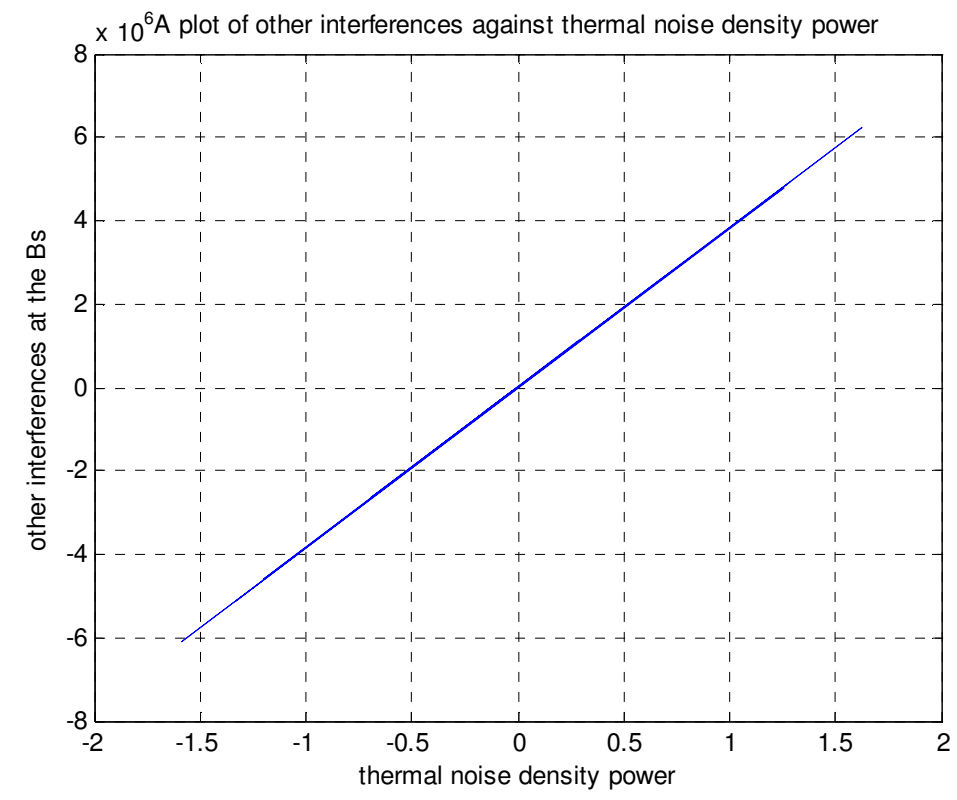

Figure 5 Relationship between interference from other cells and the thermal noise density. 
International Journal of Computer Networks \& Communications (IJCNC) Vol.4, No.5, September 2012

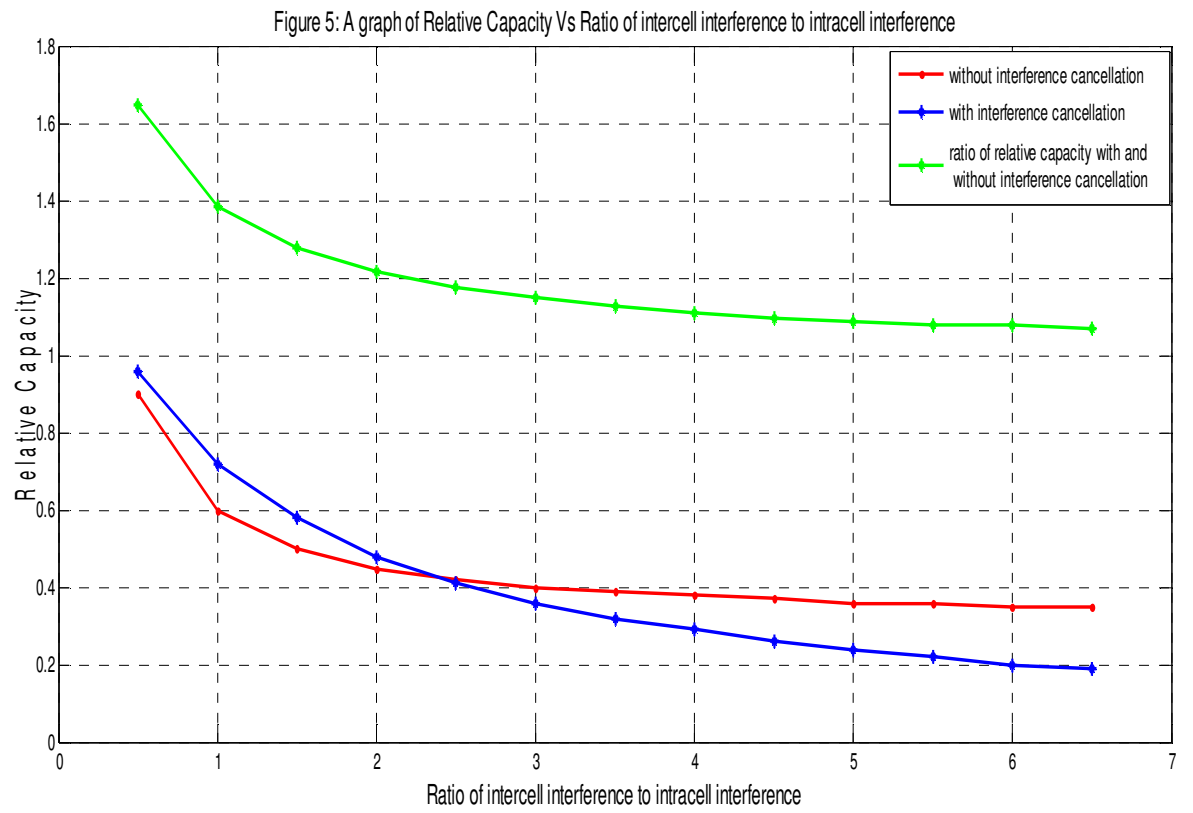

Figure 6. Plot of relative capacity and the ratio of intercell interference to intracell interference.

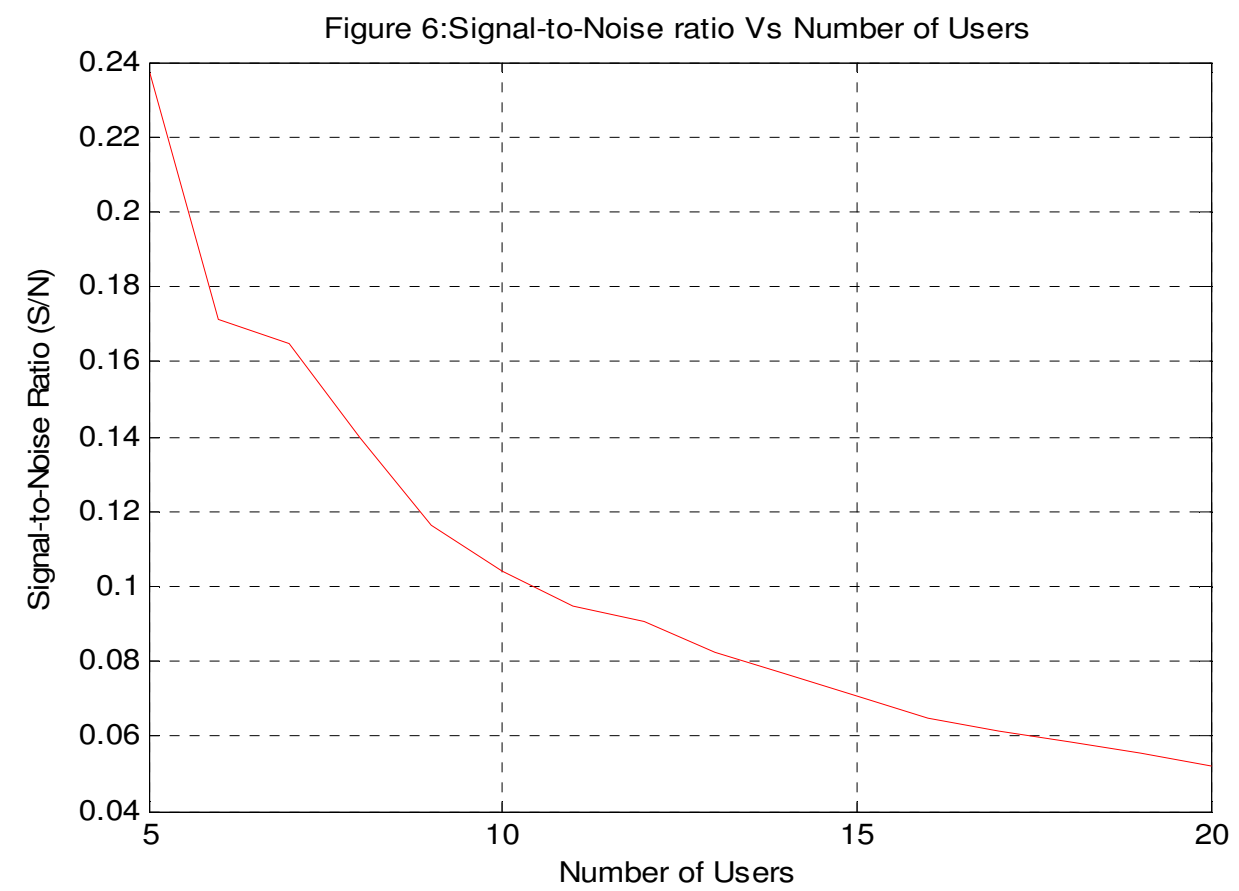

Figure 7: The relationship between signal to noise ratio and number of users. 


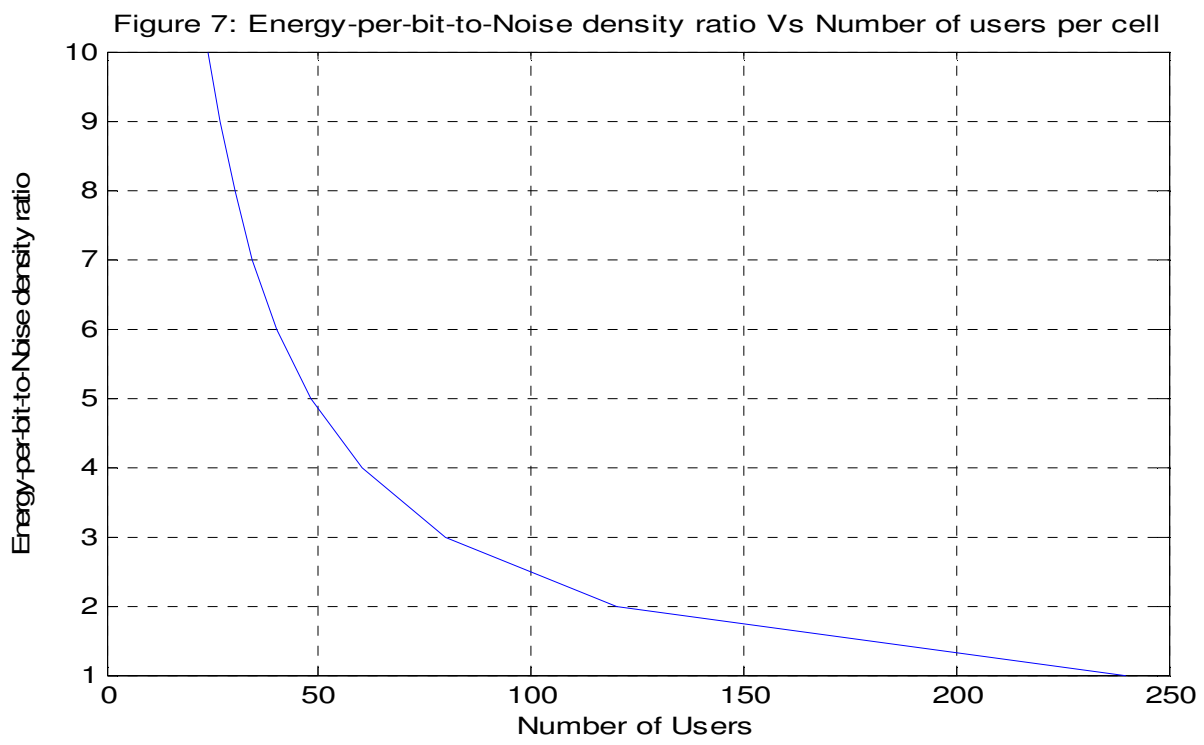

Figure 8: Showing the relationship between $\mathrm{E}_{0} / \mathrm{N}_{\mathrm{o}}$ and number or users

\section{CONCLUSION}

CDMA is interference limited and therefore any reduction in the interference will cause a linear increase in the capacity of CDMA systems. CDMA systems are usually affected by co-channel interference (CCI) and adjacent channel interference (ACI). But the ACI is more pronounced in a CDMA system. Ideal synchronization is not necessarily a prerequisite to obtaining the maximum capacity in a TD-CDMA based DCA network. Timeslot opposing method can also be used to obtain low interference in a TDD system compared with an equivalent FDD system. The base station separation distance affects the relative capacities of the CDMA- based Dynamic Channel Assignment system. The number of users in a network affects the total interference experienced; that is the larger the number of users, the higher the interference experienced and the lower the Signal-to-noise ratio. The greater the distance, the lower the ACI from other BSs which translates into high quality of service for the network users.

\section{REFERENCES}

[1] Mosa Ali Abu-Rgheff, (2007), "Introduction to CDMA Wireless communication" Academic Press, published by Elsevier Ltd, California, USA.

[2] William C. Y. Lee, (2008), “Mobile communications Engineering”, Mc Graw- Hill, New Delhi, India.

[3] John. S. Davis II, (1995), "Wireless Communication”, U.C. Berkeley Publishers 1995, London.

[4] Cox D.C and Reudink, D.O, (1991), "Dynamic Channel Assignment in High-capacity Mobile Communications Systems”, Bell System Technical Journal, Vol. 50. No.6, pp 74-97.

[5] Idigo V.E, Akpado K.A and Nsionu I.I., (2006), "Estimating the Capacity of a Multi-cell System Using Concentric Circle Geometry", International Journal of Electrical \& Telecommunication Systems Research Vol. 1 No 1, pSp19-22. 
International Journal of Computer Networks \& Communications (IJCNC) Vol.4, No.5, September 2012

[6] Harald Haas, (2000) "Interference Analysis of and Dynamic Channel Assignment Algorithms in TDCDMA/TDD Systems" The University of Edinburgh, England.

[7] Rappaport, T.S, and Milstein L.B, (1992), "Effects of Radio propagation path loss on DS- CDMA cellular Frequency Re-use Efficiency for the Reverse Channel", IEEE Transaction on Vehicular Technology, Vol. 41 No. 3, pp 231-242.

[8] A.J. Vertibi and A.M. Vertibi,(1994), "Other- Cell interference in Cellular Power-controlled CDMA", IEEE Transaction on Communications, Vol. 42, Pp.1501-1504.

[9] Ian Lim, Chedd Marley, and Jorge Kitazuru, "MIMO Z Channel Interference Management", International Journal of Computer Nteworks and Communications (IJCNC) Vol.4, No.1. January 2012. Pp. 27-38.

[10] Jon W. Mark and Weihua Zhuang, (2006) "Wireless Communications and Networking", Prentice Hall Publishers Ltd India.

[11] A. Vertibi, (1995), "Principles of Spread spectrum communication", Addison - Wesley Publishers, London.

[12] A.j. Vertibi, (1995), "Performance Limits of Error - correcting coding in Multi-cellular CDMA System with and without Interference Cancellation in Code Division Multiple Access Communications System”, Khiwer Academic Press India, Pp. 47-52.

[13] S. Hamalainen, A. Toskala, and Laukkanen, (1997), "Analysis of CDMA Downlink capacity Enhancements", Proceedings of the 1997 IEEE International Symposium on Communications PIMRC 97, Vol. 1 No. 3, Helsinki Publishers Finland, Pp-241-245.

[14] K.S. Gilhousen, I.M. Jacobss, R.Padovani, A.J. Vertibi,L.A. WSeaver,Jr, and C.E Wheatly III, (1991), "On the capacity of a Cellular CDMA System", IEEE Transaction on vehicular Technology, Vol.40 Pp.303-312.

[15] ETSi, (1998), "Universal Mobile Telecommunication system (UMTS): Selection Procedures for the Choice of Radio Transmission Technologies of UMTS" Vol. 30 No.3, TR 101 112,

\section{Authors Profile}

- Ohaneme Cletus Ogbonna is presently a lecturer in the department of Electronic and Computer Engineering of Nnamdi Azikiwe University, Awka, Nigeria. He holds $\mathrm{PhD}$ degree in Electrical/Electronic Engineering (Communication Engineering option) from the Department of Electrical/Electronic Engineering of Enugu State University of Science \& Technology (ESUT) Enugu, Nigeria. E-mail: engrohaneme@ yahoo.com

- Idigo Victor Eze is an Associate Professor of Communication Engineering and currently a lecturer in the Department of Electronic and Computer Engineering, Nnamdi Azikiwe University, Awka, Nigeria. E-mail: viceze2006@yahoo.com

- Nnebe Scholastica Uchenna is currently a lecturer in the department of Electronic and Computer Engineering of Nnamdi Azikiwe University Awka, Nigeria. She is currently a Ph.D student of Communication Engineering in the same department and of the same institution. Email: scholar.nnebe@gmail.com

- Ifeagwu Emmanuel Ncheta is a lecturer in the department of Electronic and Computer Engineering of Nnamdi Azikiwe University Awka, Nigeria. She is currently a Ph.D student of Communication Engineering at Department of Electrical/Electronic Engineering of Enugu State University of Science and Technology, Enugu. E-mail: skotolysis@ yahoo.com 